\title{
DESIGN AND FABRICATION OF SOLAR POWERED ICE BOX
}

\author{
Arpit $^{1}$, Vivek Verma ${ }^{2}$ \\ ${ }^{I}$ Mechanical and Automation Department, Amity University, Lucknow \\ ${ }^{2}$ Assistant Professor, Mechanical and Automation Department, Amity University, Lucknow
}

\begin{abstract}
This paper describes design and fabrication of solar powered icebox that uses solar power for the power and in this system the compressor is replaced with thePeltier module to produce desired cooling, which makes it more efficient and compact. The basic idea of the system is affordable, portable, consume less power and gives the same amount of cooling effect as that of conventional icebox. The system provides satisfactory results. The only disadvantage observed is; long time required for desired cooling effect.
\end{abstract}

Keywords: Solar power, Peltier Module.

\section{INTRODUCTION}

An icebox is a compact non-mechanical refrigerator, which was a common kitchen appliance before the development of safe powered refrigeration devices. This system does not have any compressor to compress the refrigerant, which makes it more compact, portable, consumes less power and small in size. In addition the conventional ice-box are not portable and cannot be carried easily while travelling or going for the picnic but the solar powered ice-box can easily carried while going for the picnic or going for a poolside party as solar powered uses solar energy.

This paper conceptualizes an alternative designmethod called solar powered icebox. It is a low cost, affordable and homemade system produces satisfactory result. This low cost system can havewide range of applications in urban as well as rural areas.

\section{SYSTEM DESCRIPTION}

In this system, a copper box of $20 * 20 \mathrm{~cm}$ is wrapped up with the copper tubing in which the refrigerant (i.e., water) is filled and the two ends of the copper tubing are attached with water block CPU cooler with the help of M-seal so that the refrigerant cannot leak from the joint. A Peltier module is attached on the water block CPU cooler whose cooling side is attached to the surface of the water block CPU cooler and the heating face is attached with heat sink, which is releasing heat to the atmosphere, and the whole assembly is made insulated with the help of thermocol. The whole assembly is placed in a wooden box of $50 * 50 \mathrm{~cm}$. the solar panels are attached on the four faces of the wooden box and the solar panels are attached on the wooden box with the help of chain.

- $* * *$

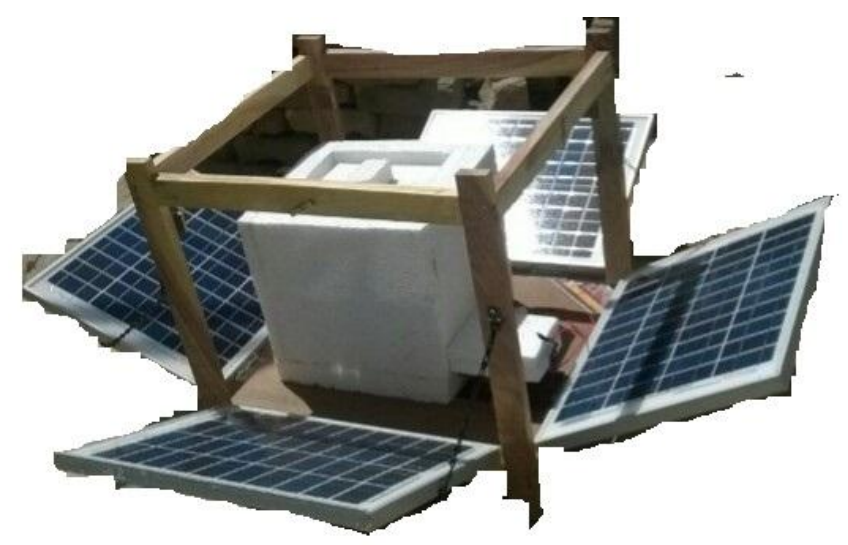

Figure 3: Layout of Solar Powered Icebox

\section{FABRICATION}

a) Cooling Unit

Cooling unit consist of a Peltiermodule, which is sandwich between the water block CPU cooler, and the heat sink. The Peltier module is sticked on the heat sink with the help of cooling paste and the whole assembly is attached on the water block CPU cooler with the help of wire. The heat sink attached to the hot junction of the Peltier module takes the heat of the Peltier plate and gives it to the environment with the help of CPU fan.

\section{b) Condensing Unit}

Condensing unit consists of the cooling tube in which the refrigerant is cooled and condensed and the water block CPU cooler. The refrigerant, while passing through the water block CPU cooler, gives up its latent heat to the surrounding condensing medium, which is normally air or water. Water block CPU cooler input and output ends are joined with the copper tubes by the help of m-seal. Both the ends of the water block CPU cooler are fixed tightly so that the refrigerant cannot leak. 


\section{c) Evaporative Unit}

Evaporative unit in the solar powered icebox system is the copper box. The function of an evaporator is to absorb heat from the surrounding location or medium, which is to be cooled, by means of a refrigerant. The temperature of the refrigerant in evaporator must be less than that of the surrounding medium so that the heat flows to the refrigerant. The evaporator becomes cold and remains cold due to the following two reasons:

1. The temperature of the evaporator coil is low due to the low temperature of the refrigerant inside the coil.

2. The low temperature of the refrigerant remains unchanged because any heat it absorbs is converted to latent heat as heating proceeds.

\section{d) Solar Power}

The specialty of this system is: it is powered with solar power. Due to the use of solar energy, the efficiency of the system is infinite as there is no capital for the input power is required. Solar energy is a renewable source of energy. Solar power is the conversion of sunlight into electricity, either directly using photovoltaics (PV), or indirectly using concentrated solar power (CSP). Concentrated solar power systems use lenses or mirrors and tracking systems to focus a large area of sunlight into a small beam. Photovoltaics convert light into an electric current using the photovoltaic effect.

Photovoltaic were initially solely used as a source of electricity for small and medium sized applications, from the calculator powered by a single solar cell to remote homes powered by an off grid rooftop PV system. As the cost of solar electricity has fallen, the number of grid connected solar PV systems has grown into the millions and utility scale solar power stations with hundreds of megawatts are being built. Solar PV is rapidly becoming an inexpensive, low carbon technology to harness renewable energy from the Sun

\section{WORKING}

The solar powered icebox based on the principle of the Peltier effect which states that Peltier effect is the presence of heating or cooling at an electrified junction of two different conductors. When a current is made to flow through a junction between two conductors $\mathrm{A}$ and $\mathrm{B}$, heat may be generated (or removed) at the junction. The Peltier effect is the reverse of the Seeback effect, which states that the Seeback effect is a phenomenon in which a temperature difference between two dissimilar electrical conductor and semiconductors producing a voltage difference between the two substances. Here we use the above concept and produce desired refrigeration. The sunrays are allowed to fall on the solar panel, which are adjusted at such angle so that the maximum rays from the sun may fall on the panel. The outer casing in which all the solar panel are adjusted to such a angle so that the maximum of incident rays can fall on this by means of a chain which is fitted to a wooden block i.e. outer casing of the solar powered ice-box. The incident rays falling on the solar panel from the sun excite the electrons of the solar cell, which are arranged together in the form of a solar panel, thus producing D.C. electric current. Solar panel refers to a panel designed to absorb the sunrays as a source of energy for generating electricity. A photovoltaic (PV) module is a packaged, connected assembly of $6 * 10$ solar cells. Solar photovoltaic panel constitutes the solar array of a photovoltaic system that generates and supply solar electricity in applications. Solar modules use light energy (photons) from the sun to generate electricity through the photovoltaic effect. The majority of modules use wafer based crystalline silicon cells based on cadmium telluride or silicon. Electrical conductors are made in series to achieve a desired output voltage and in parallel to provide to a desired current capability. The conducting wires that take the current off the module may contain silver, copper or other non-magnetic conductive metal. The cells must be connected electrically to one another and to the rest of the system and thus producing the D.C. electric current at the output. With the help of the wires, the D.C. current produced at the output of the solar panel is thus transmitted to Peltier module, results in creating a hot junction and cold junction respectively at the two different conductors. The heat generated at the hot junction is emitted to atmosphere with the help of heat sink and fan setup. On the application of transfer of D.C. electric current to the Peltier module the one junction which is at hot terminal is attached with the heat sink and further with the fan setup by the medium of cooling paste. The main function of heat sink is that to take heat from the hot junction of the Peltier module and emit it to the atmosphere by the means of fan setup so that all the generated heat at the junction can be emitted to atmosphere and the required cooling effect is experienced inside the icebox effectively. The fan is operated by the D.C. current generated by solar panel. Thus, the entire setup aids in emitting out the heat generated during the process to the atmosphere.

The cooling effect produced on the opposite junction of Peltier module by the transmission of D.C current from the output of the solar panel to the Peltier module is transmitted to water block CPU cooler by directly attaching the cold junction surface of the Peltier module to the surface of water block CPU cooler. The purpose of using additional water block CPU cooler is to provide maximum surface area for the contact of the cold junction of the Peltier module and to the refrigerant (fluid) which transfers the rest of the cooling effect more effectively. The cooling thus produced on the surface of water block CPU cooler is transferred to the refrigerant inside the copper tubing surrounding the core of icebox, thus helps in giving faster cooling effect inside the core icebox with respect to the time.

Since copper tubing is surrounding the maximum surface of the core ice-box and is welded in circumferential form to all of the surface of core ice-box and the both ends of the copper tubing surrounding the core ice-box is attached to the both the opening of the water block CPU cooler, thus the 
cooling effect produced within the copper tubes is transmitted to the walls of the box which were surrounded by the copper tubing by phenomenon of conduction, and thus providing the required refrigeration effect inside the core ice-box.

A proper insulation by means of Expanded Polystyrene (Thermocol) is done on the outer surface of core icebox; so that the refrigeration effect produced in the copper tubing does not escape to the atmosphere. In order to make the core box properly insulated two to three layers of Expanded Polystyrene (Thermocol) are attached together, and finally attached to the core icebox, which was pre-insulated by a thin layer of Expanded Polystyrene (Thermocol). The Expanded Polystyrene (Thermocol) of thin layer in the small sizes according to the surface area covered by the copper tubing to the core ice-box is attached between the ice-box and the core of ice-box so that the cooling effect from the copper tubing transmit only towards the core of ice-box and all the area except around the contact between the copper tubing and the core ice-box is insulated by these thin layers of Expanded Polystyrene (Thermocol). To make it better insulating chamber two-three thick layers of the Expanded Polystyrene (Thermocol) is further attached to the each layer with respect to pre-attached layer by medium of fevicol.

Lid of core icebox is made separately with the help of insulating medium i.e. Expanded Polystyrene (Thermocol) and aluminum foil so that the desired the cooling effect remains inside the core icebox and cools the chamber instead escaping to the atmosphere.

\section{COMPONENTS}

The primary components of the refrigeration system are:

\section{a) Copper box}

Copper a soft, malleable and ductile metal with very high thermal and electrical conductivity. In solar powered icebox is box in which cooling will be produced is made up of copper of dimension $20 * 20 \mathrm{~cm}$.

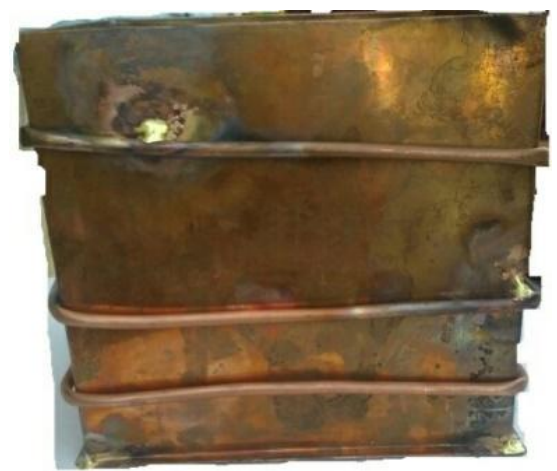

Fig.2 Copper box

\section{b) Copper tube (Heat Exchanger)}

Copper a soft, malleable and ductile metal with very high thermal and electrical conductivity. It is used as a conductor of heat and electricity, as a building material, and as a constituent of various metal alloys. The copper tube is wrapped around the copper box and used as a heat exchanger between the box and the refrigerant. This ensures greater efficiency during heat transfer between the tube and the box.

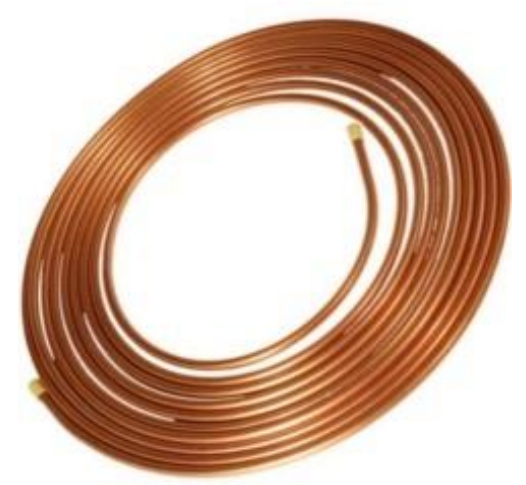

Fig.3 Copper Wire

\section{c) Water Block CPU cooler}

A water block CPU cooler is a type of heat sink that cools and dispenses water. It works as a medium to transmit the cooling produced by the Peltier module to the refrigerant inside the copper tubing.

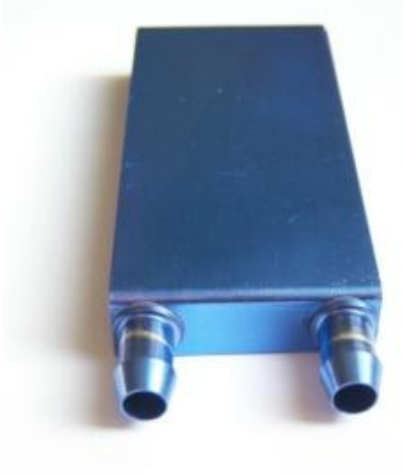

Fig.4 Water block CPUcooler

\section{d) Refrigerant}

A refrigerant is a substance or mixture, usually a fluid, used in a heat pump and refrigeration cycle. In most cycles, it undergoes phase transitions from a liquid to a gas and back again. In this system, we used water as the refrigerant because of the high heat transfer rate of the water.

\section{e) Peltier Plate}

Thermoelectric cooling uses thePeltier effect to create a heat flux between the junctions of two different types of materials. A Peltier cooler, heater, or thermoelectric heat pump is a solid-state active heat pump, which transfers heat from one side of the device to the other, with consumption of electrical energy, depending on the direction of the current. Such an instrument is also called a Peltierdevice. It can be used either for heating or for cooling, although in practice the main application is 
cooling. Its main disadvantage is high cost and poor power efficiency. The device has two sides, and when DC electricity flows through the device, it brings heat from one side to the other, so that one side gets cooler while the other gets hotter. The "hot" side is attached to a heat sink so that it remains at ambient temperature, while the cool side goes below room temperature.

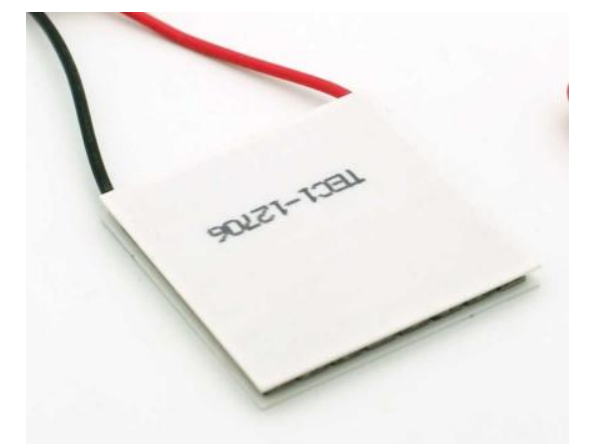

Fig.5 Peltier module

\section{f) Heat Sink}

A heat sink is a passive heat exchanger that transfers the heat generated by an electronic or a mechanical device into a coolant fluid in motion. Then-transferred heat leaves the device with the fluid in motion, therefore allowing the regulation of the device temperature at physically feasible levels. A heat sink is designed to maximize its surface area in contact with the cooling medium surrounding it, such as the air. Air velocity, choice of material, protrusion design and surface treatment are factors that affect the performance of a heat sink. A heat sink is usually made out of copper and/or aluminum. Aluminum is used in applications where weight is a big concern.

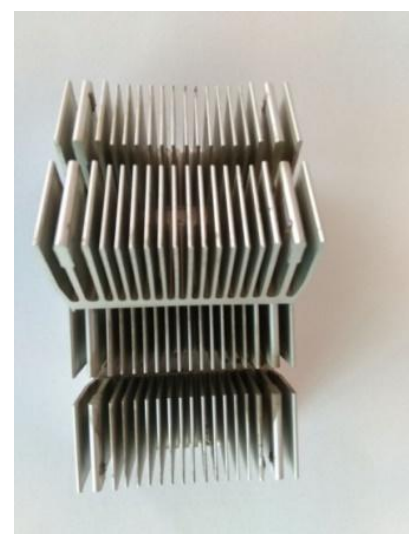

Fig.6 Heat sink

\section{g) Insulation to ice-box}

In this system the insulation material used is expanded polystyrene (Thermocol)offers a non-hydroscopic, odorless, rigid, closed cell Expanded Polystyrene containing $98 \%$ by its volume still air entrapped in its cell and is the major reason for its excellent insulation properties. The insulation material is wrapped over the box, which decreases the heat losses.

\section{Applications of insulation:}

- Commercial Air-conditioning

- Residential Air-conditioning

- Air-conditioning in Industrial houses

- Cold Storages

- Mushroom plants

- Milk Plants

- Sound proofing

- False Ceiling etc.

\section{h) Solar panel}

As the name of the system suggest, the power to the solar powered icebox has been provided by the solar energy, which is a renewable source of energy. The Solar panel refers to a panel designed to absorb the sun's rays as a source of energy for generating electricity or heating.

Solar Photovoltaic panels constitute the solar array of a photovoltaic system that generates and supplies solar electricity in commercial and residential applications. Each module is rated by its DC output power under standard test conditions, and typically ranges from 100 to 365 watts. In this system, four panels each of 25-watt output power are used.

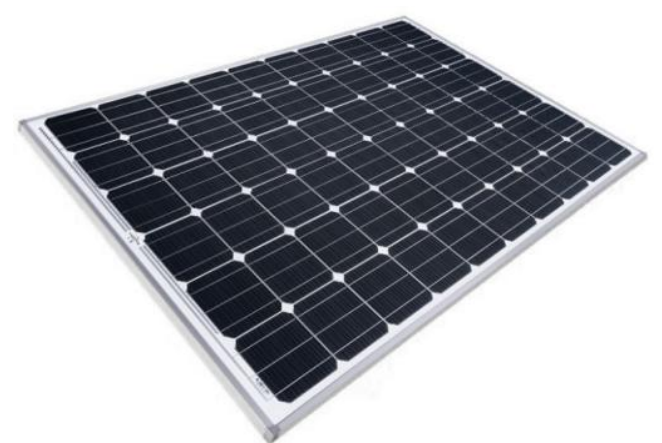

Fig.7 Solar Panel

\section{i) Wires}

A wire is a single, cylindrical flexible strand or rod of metal. Wires are used to bear mechanical loads or electricity and telecommunication signals. Wire is commonly formed by drawing the metal through a hole in a die. Wire comes in solid core, stranded, or braided forms.

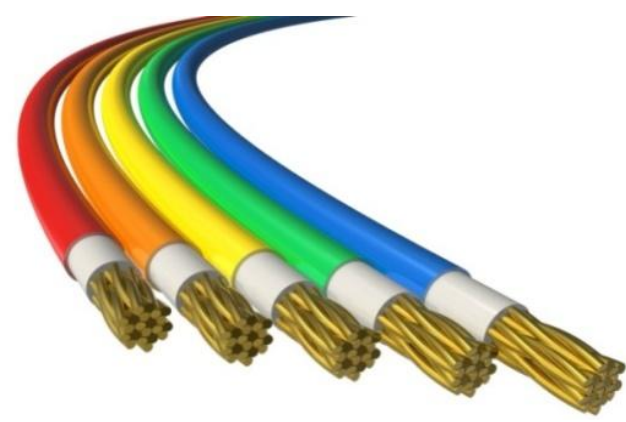

Fig.8 Wires 


\section{OBSERVATION}

\begin{tabular}{|l|l|l|}
\hline S. No. & Time $(\mathbf{m i n})$ & Drop in Temperature $\left({ }^{\circ} \mathbf{C}\right)$ \\
\hline 1 & 3 & 0.5 \\
\hline 2 & 6 & 1.2 \\
\hline 3 & 9 & 4 \\
\hline 4 & 12 & 6 \\
\hline 5 & 15 & 8 \\
\hline 6 & 18 & 12 \\
\hline 7 & 21 & 15 \\
\hline
\end{tabular}

\section{GRAPH}

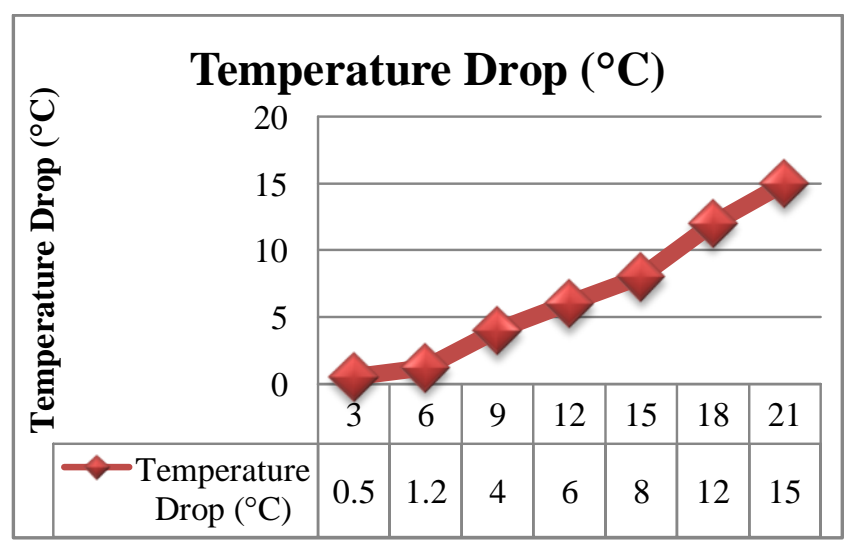

\section{CONCLUSION}

Solar powered icebox promises dramatic reduction in electricity reduction of the iceboxes as well as refrigeration systems. The efficiency of the solar powered icebox is infinite as there is no cost for the input power as the solar energy is a free as well as renewable source of energy. Solar energy is the future of the power consumption.

Solar powered icebox is simple to manufacture and it can be carried easily while travelling and while going for a party outdoor or a picnic. It is portable, affordable.

\section{REFERENCES}

[1]. Green, M. (2000) Power to the People: Sunlight to Electricity Using Solar Cells, New South Wales University Press.

[2]. Chapin, D., Fuller, C. and Pearson, G. (2004) A new silicon $p-n$ junction photocell for converting solar radiation into electrical power, J. Appl. Phys. 25, 676

[3]. Steve Leone (9 December 2011). "Report Projects Massive Solar Growth in India". Renewable Energy World.

[4]. Muneer, T,; Asif, M.; Munawwar, S. (2005). "Sustainable production of solar electricity with particular reference to the Indian economy". Renewable and Sustainable Energy Reviews 9 (5): 444. Doi:10.1016/j.rser.2004.03.004. edit (publication archived in ScienceDirect, needs subscription or access via university).

[5]. "Status of Solar Energy in INDIA - 2010". Retrieved 2010-11-27.

[6]. Rowe D. M., 1995, CRC handbook of Thermoelectrics.
[7]. Huang B.J., Chin C.J. \& Duang C.L., 2000, “A design method of thermoelectric cooler", International Journal of Refrigeration, 23: 208-218.

[8]. Xi Hongxia, Luo Lingai and Fraisse Gilles, 2007, "Development and applications of solar-based thermoelectric technologies", Renewable and Sustainable Energy Reviews, 11(5): 923-936.

[9]. Dai Y.J., Wang R.Z. and L. Ni, 2003, "Experimental investigation and analysis on a thermoelectric refrigerator driven by solar cells", Solar energy material and solar cells 77:377-391.

[10].Gao Min and Rowe D.M., 2006, "Experimental evaluation of prototype thermoelectric domesticrefrigerators", Applied Energy, 83 (2): 133-152.

[11].Bansal P.K. and Martin A., 2000, "Comparative study of vapour compression, thermoelectric and absorption refrigerators", International Journal of Energy Research, 24: 93-107.

[12].Astrain D and Vian J G (2005), "Computational Model for Refrigerators Based on Peltier Effect Application", Applied Thermal Engineering, Vol. 25, No. 13, pp. 3149-3162.

[13].Christian J L and Jadar R Barbosa Jr (2011)," Thermodynamic Comparison of Peltier, Stirling, and Vapour Compression Portable Coolers", Applied Energy, Vol. 9, pp. 51-58.

[14].Taylor, R.A., Solbrekken, G., Comprehensive systemlevel optimization of thermoelectric devices for electronic cooling applications, Components and Packaging Technologies, IEEE Transactions on (Volume:31, Issue: 1)

[15]."Thermoelectric cooling", [Online]. Available: www.wikipedia.org.

[16].En.wikipedia.org/Thermo Electric Effect.

[17].G.J. Vella L.B. Harris, and H.J. Goldsmid, "A solar thermoelectric refrigerator", Solar Energy, Vol. 18, pp. 355-359, 1976

[18].Sofrata, H., 1984. "Solar Thermoelectric Cooling System". In: S.W. James, B.H. Khoshaim, R. Mallory and A. Meiners, Editors: Solar Buildings. Missouri: Midwest Research Institute, pp: 59-76.

[19].www.tetech.com 